Pausan, N., Liu, Y., Lu, Y. and Hutchinson, AR. (2016) 'The use of expandable graphite as a disbonding agent in structural adhesive joints', Journal of Adhesion

DOI: https://doi.org/10.1080/00218464.2016.1226169

This document is the authors' Accepted Manuscript.

License: https://creativecommons.org/licenses/by-nc-nd/4.0

Available from RADAR: https://radar.brookes.ac.uk/radar/items/0f79aa51-7dbf-425b-a6fb-04d8e2eb0e08/1/

Copyright $(\subseteq$ and Moral Rights are retained by the author(s) and/ or other copyright owners unless otherwise waved in a license stated or linked to above. A copy can be downloaded for personal non-commercial research or study, without prior permission or charge. This item cannot be reproduced or quoted extensively from without first obtaining permission in writing from the copyright holder(s). The content must not be changed in any way or sold commercially in any format or medium without the formal permission of the copyright holders. 


\section{The use of expandable graphite as a disbonding agent in structural adhesive joints}

Pausan, N, Lui, Y, Lu, Y and Hutchinson, AR.

\section{Abstract}

Expandable graphite offers the potential to be used as a controllable adhesive disbonding mechanism in an adhesively bonded joint. The graphite particles can be incorporated, in small quantities, into the adhesive layer. Heat can be used to trigger the expansion and thereby facilitate disassembly of structurally bonded assemblies. An experimental programme was developed to investigate the engineering capabilities of different types of bonded joints, made with an epoxy and a polyurethane adhesive, containing different amounts of expandable graphite. The short- and long-term mechanical properties of lap shear and wedge cleavage joints were evaluated. In contrast to the incorporation of more conventional physical and chemical foaming agents into bondlines, typically in excess of a $10 \%$ addition level, it was found that the addition of just a few percent of graphite was sufficient to provide a reliable disbonding mechanism. Moreover, the usual adverse side-effects on joint performance, associated with incorporating functional additives, were far more limited.

\section{Keywords}

Disbonding; graphite; epoxy; polyurethane; aluminium; lap shear; wedge cleavage; durability 


\section{Introduction}

The ability to be able to separate adhesive bonded assemblies, without causing damage to the substrates, is very desirable in order to allow the recovery and reuse of high value materials. This may be for repositioning in manufacturing, repair in service, or recovery of materials at end-of-life. Various methods for adhesive reversibility or disbonding have been proposed over the last 30 years but there currently exist no universally accepted solutions for disbond-on-demand bonded joint applications.

The disbonding methods that have been developed fall into three main categories: (1) reworkable adhesive systems, (2) electrically reversible adhesives and (3) the incorporation of functional additives into the adhesive [1]. For structural applications of adhesives, the latter method offers the most promise but there are many factors to be taken into consideration for a whole systems approach [2]. Functional additives all employ the principle of expanding the adhesive bondline, or more effectively a primer layer, using an energy source to stimulate the additive and create gas evolution. Several papers on this topic have examined physical foaming agents [3], or microspheres [4], and chemical foaming agents [5, 6]. Naturally, the volumetric expansion needs to be sufficient to overcome the adhesive's ability to absorb the expansion. Thus, a balance needs to be found between expansion potential, in terms of type and amount of additive, and the adhesive's toughness. For example, it is typically easier to expand an epoxy bondline than it is to expand a relatively tough polyurethane bondline, because polyurethane can absorb more deformation and strain energy [7]. 
A further consideration is the amount of energy, or heat, that is required to trigger the mechanism. Additives may be chosen that begin to work at temperatures below $100^{\circ} \mathrm{C}$, but this may compromise both the adhesive bonding cure process and the upper temperature service limit of the bonded product. Again, the additive needs to be chosen carefully with assembly and service requirements in mind.

The inclusion of an additive to a formulated adhesive, even in small amounts, has the potential to change the application characteristics and the mechanical performance characteristics in service. The mechanical performance issues include changes to the cured adhesives physical properties such as modulus, strength, strain to failure, fracture toughness and glass transition temperature. Banea et al [8] discussed adhesive material reformulation to balance additive inclusion and preservation of toughness. Lu et al. [3] proposed a method to improve the bond stability between the surface of microsphere additives and the adhesive matrix, thereby maintaining matrix stability and long-term joint performance. Most of the previous research reported on microsphere additions has highlighted significant changes to the short- and long-term performance of bonded joints $[3,8,9]$. The significant problems include drastic loss of environmental durability and loss of joint fracture toughness.

Kishi et al. [10,11] reported problems with triggering the expansion of microspheres at temperatures as low as $60^{\circ} \mathrm{C}$. They therefore proposed the use of expanding graphite as an additive to provide higher temperature bonded joint performance for industries such as automotive. The authors discussed the development of matrix resin formulations to incorporate 
graphite and then showed the positive short-term results on dismantlability using a $10 \%$ addition level.

Expandable Graphite (EG) is a form of graphite oxide intercalation compound that exfoliates when heated. The compound can be made from natural graphite flakes (the natural flaky structure of graphite is shown in Fig. 1) with chemical $[12,13]$ or electrochemical $[14,15]$ intercalation processing. Once triggered by direct heating or microwave-assisted heating $[16,17]$, the latent intercalant (e.g. sulphuric/nitric acid anions) "trapped" in the EG become instantly activated which decompose into dioxide/trioxide gas and water, causing the graphite flakes to expand up to hundreds of times thicker than the original graphite [18] (Fig. 2), creating an accordion-like structure (Fig. 3). Details of the microscopy procedures used to generate Figures 1 and 3 are provided in section 2.2.5.

EG is chemically inert and has comparable thermal and electrical conductivity to graphite $[19,20]$. It has been widely adopted for packaging and insulation $[20,21]$, as fire retardants [22-25], as gasketing and supporting materials [26, 27] and for producing graphene [16, 28] and batteries [29-32]. EG has also been used as additives or filler materials in composites [33, 34], pressure sensitive adhesives, sealants and coatings.

Due to its inherent features, EG represents an interesting layered material whose high expansivity makes it an attractive foaming agent for active disbondable adhesives. This paper reports the results of a parametric experimental programme to investigate the engineering capabilities of different types of bonded joints, made with an epoxy and a polyurethane 
adhesive, containing different amounts of expandable graphite. The shortand long-term mechanical properties of bonded joints were evaluated.

\section{Experimental programme}

The experimental programme followed a structured progression of evaluating bonded joint characteristics (Fig. 4). Preliminary investigations of additive type and amount were informed by previous research $[3,5]$, leading to an efficient experimental approach. The adhesives and substrates used were chosen with relevance to automotive industry applications. This enabled the results to be compared directly with our previous research on the mechanical performance of joints made with expanding microspheres.

EG samples were first expanded with heat in glass beakers in order to provide a guide to the activation temperature and some visual assessment of expansion potential. The expanded particles were then examined using SEM. These experiments enabled us to select the range of addition levels for evaluation.

\subsection{Materials}

\subsubsection{Adhesives}

Two types of adhesives were selected for this study, polyurethane (PU) SikaForce 7888 L10 supplied by Sika and epoxy Scotch-Weld ${ }^{\text {TM }}$ EC-9323 supplied by $3 \mathrm{M}$. Both are two component $(2 \mathrm{~K})$, moderate temperature-cured, adhesives used for structural or semi-structural applications. Scotch-Weld 9323 is a high performance epoxy used for joining low, medium and high 
volume production vehicles. It is also used in the motorsports industry because of its high shear strength, good durability, high impact resistance and ability to join metals and polymer composites [5]. SikaForce 7888 is a high strength, fast-cure polyurethane adhesive which can be cured at room temperature. It was selected to represent a family of PU based adhesives which are becoming popular in recent years for their good resistance to peel, high elongation at break, improved impact resistance and fatigue performance [7]. The general mechanical properties of the PU and epoxy adhesive are given in Table 1.

\subsubsection{Substrates}

Aluminium alloy EN AW-6082 T6, a high strength structural aluminium alloy, was selected for all experimental testing. The tensile properties of the material are given in Table 2. Different gauges and specifications were selected for the lap-shear and wedge cleavage specimens according to the different characteristics required for each test joint type. For single lap-shear joints (SLJs), $2 \mathrm{~mm}$ sheet aluminium was used while $5 \mathrm{~mm}$ thick aluminium adherends were selected for the wedge tests to avoid plastic deformation.

\subsubsection{Expandable Graphite}

The EG used was SYZR 501, which is a low sulphur model manufactured by SanyoCorp, Japan. SYZR 501 is designed for a flame retardant system but can also be used in sealants, coatings and polymers. The initial volume is 
$0.44 \mathrm{ml} / \mathrm{g}$ but the final expansion volume can reach more than $230 \mathrm{ml} / \mathrm{g}, 500$ times higher than the initial volume. The starting expansion temperature is $150^{\circ} \mathrm{C}$ and the expansion volume against temperature is shown in Figure 5.

\subsection{Test Methods}

\subsubsection{Single Lap-Shear Tests}

Single lap-shear joint (SLJ) tests were conducted with reference to BS EN $1465: 2009$ and the geometry of the test specimens is depicted in Fig.6. SLJs were assembled with pre-cut aluminium sheets using pure and EG-modified PU and epoxy adhesives. The EG was added to the adhesive in different amounts, referred to an percentage additions by weight (\%wt). An asymmetric centrifugal mixer, SpeedMixer DAC150-FVR-K, was used to mix the adhesive with EG at $3000 \mathrm{rev} / \mathrm{min}$ for 60 s. Before applying the adhesives, the substrate surfaces were abraded with Scotch-Brite pads and an acetone degrease. $0.5 \%$ wt solid glass spheres (Ballotini) were added to the adhesive to control bondline thickness at $0.2 \mathrm{~mm}$. Joints made with both types of adhesive were cured at $90{ }^{\circ} \mathrm{C}$ for 90 minutes and left at room temperature for 7 days. An Instron 5582 universal test machine equipped with a $100 \mathrm{kN}$ load cell was used for the tensile testing at $1 \mathrm{~mm} / \mathrm{min}$ quasi-static load. The test environment was $23 \pm 4{ }^{\circ} \mathrm{C}$ and $30 \pm 5 \%$ relative humidity. Typically, 5 or 6 replicate joints were tested for each variable. 


\subsubsection{Disbonding Effectiveness Test}

SLJ specimens were used to examine the effectiveness of the EG as a disbonding agent in the adhesive. A high temperature oven was used to heat up specimens from room temperature to $235^{\circ} \mathrm{C}$ and was kept for 1 minute to ensure uniform thermal distribution throughout the bondline. If the anticipated total disbonding did not take place, the joints were tested for residual lapshear strength. The disbonding effectiveness was then assessed by comparing the tensile lap-shear shear strength of joints before $\left(\tau_{\mathrm{SL}}\right)$ and after $\left(\tau^{\prime} \mathrm{SL}\right)$ heat activation, which can be expressed by eqn.1:

$$
E_{\text {disb }}=\frac{\tau_{S L J \_} \tau^{\prime} S L J}{\tau_{S L J}}
$$

SLJs made using pure adhesives were also tested for strength for two main reasons: (a) to generate control data as reference for investigating SLJs bonded with adhesive I EG matrices, and (b) to determine the effect of elevated temperature exposure on the adhesive and, subsequently, on the joint strength.

\subsubsection{Environmental Conditioning Test}

A simple water immersion test was used to evaluate the performance of the epoxy adhesive bonded joints with/without EG added to the bondline when subjected to hydrothermal ageing. SLJ specimens were immersed in clean water at a constant temperature of $55^{\circ} \mathrm{C}$ for 90 days prior to testing. 


\subsubsection{Wedge Cleavage Tests}

The wedge test, originally developed by Boeing (ASTM D 3762-03), is a simple and inexpensive procedure to qualitatively assess the ability to resist crack propagation with forces and environmental effects on adhesive bonded joints. The test involves forcing a wedge into the bond-line of a flat bonded joint (Fig. 7), thus creating cleavage (peel) stresses in the adhesive bondline whilst the stressed specimen is exposed to an aqueous environment. Our objectives were to assess joint toughness and resistance to bondline crack propagation. We did not want premature adhesion failure to occur, so a rigorous surface treatment procedure was adopted. This involved Scotch-Brite abrasion and acetone degreasing of the aluminium substrates, as before, followed by the application of a silane primer. The material chosen was a $5 \%$ solution of a $y$-glycidoxypropyltrimethoxy silane in an alcohol-water solution supplied by Permabond. The bondline thickness was controlled at $0.2 \mathrm{~mm}$ using Ballotini. PTFE tape was applied at the end of the substrates to enable wedge insertion without disturbance from the adhesives. Joints made with both types of adhesive were cured at $90^{\circ} \mathrm{C}$ for 90 minutes and left at room temperature for 7 days. Wedges were inserted in the cured specimens at $30 \mathrm{~mm} / \mathrm{min}$ and the initial crack length was recorded. The specimens were then immersed in water at $55^{\circ} \mathrm{C}$ and taken out for crack length observation at various intervals. Four separate batches of joints were made: (a) pure PU adhesive, (b) pure epoxy adhesive, (c) PU adhesive with 5\%wt EG, and (d) epoxy adhesive with $3 \%$ wt EG. The crack lengths were measured using a Mondo 450 back illuminated microscope and measurements of crack 
propagation were made for each specimen at $6,24,72$, and 168 hours. A minimum of 3 joints were tested for each variable.

The fracture energy was estimated by applying linear elastic beam theory (Eqn.2). The assumption is that no yielding of the substrates is present. The formula corrects for shear distortion of the substrates, and contains experientially derived corrections for the beam rotation at the crack tip for a homogeneous specimen.

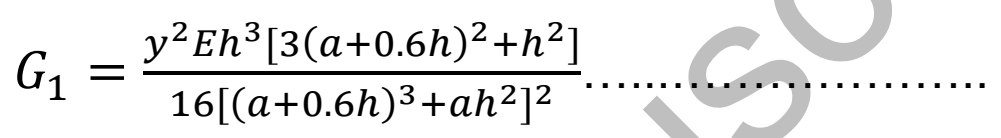

Where:

$\mathrm{G}_{1}=$ strain energy release rate $\left(\mathrm{J} / \mathrm{m}^{2}\right)$;

$\mathrm{y}=$ displacement at wedge insertion $(\mathrm{mm})$;

$\mathrm{E}=$ tensile modulus of substrate (GPa);

$\mathrm{h}=$ thickness of substrate normal to plane of bonding $(\mathrm{mm})$;

$\mathrm{a}=$ crack length $(\mathrm{mm})$.

\subsubsection{Microscopy}

A JEOL JSM-6010PLUS/LA Scanning Electron Microscope (SEM) was employed to examine graphite/EG particles and joint failure surfaces.

Graphite/EG particles and joint facture surfaces were carbon coated, and a $10 \mathrm{kV}$ beam accelerating voltage was used for clear images at $60 \mathrm{X}$ and $250 \mathrm{X}$ magnification ratios. 


\section{Experimental Results}

\subsection{Effect of Additive on Short-term Joint Properties}

\subsubsection{Effect on PU adhesive}

Three addition levels of EG by weight ( $3 \% \mathrm{wt}, 5 \% \mathrm{wt}, 15 \% \mathrm{wt})$ were added to the PU adhesive to investigate the residual strength of the joints after heat activation. Initial trials showed that $15 \%$ wt of $E G$ added to a $1 \mathrm{~mm}$ thick $\mathrm{PU}$ bondline led to complete separation of the joint after heat treatment. With the elevated temperature, a notable popping sound could be heard at $200-220^{\circ} \mathrm{C}$ which indicated that significant thermal expansion of the EG took place. EG contents of $5 \%$ wt and $3 \%$ wt were subsequently tested with a $0.2 \mathrm{~mm}$ thick bondline to investigate the effect of \%wt of EG additive on the lap shear strengths of PU bonded specimens, before and after heat treatment. It was found that complete separation of the joint was achieved with $5 \%$ wt of added EG and that the joint was distended by approximately $5 \mathrm{~mm}$ due to the extensive expansion of EG (Fig.8). The disbonding effectiveness (Eqn.1) as a function of EG content in the PU adhesive is depicted in Fig. 9. It can be seen that both initial and residual joint strength decreased with a higher \%wt of EG in the PU. It was noted that the initial strength increased by $2.5 \%$ at $3 \%$ wt of added EG, before a $10 \%$ loss occurred at $5 \%$ wt. In contrast, the residual strength decreased significantly from 5.22 MPa to only $0.6 \mathrm{MPa}$ with $3 \%$ wt EG content in the joint, and reached complete separation at 5\%wt. Therefore, it can be concluded that $3-5 \%$ wt of EG is sufficient to disbond PU bonded joints after heat treatment. 


\subsubsection{Effect on epoxy adhesive}

Three addition levels of EG by weight (1\%wt, $3 \% \mathrm{wt}, 5 \% \mathrm{wt})$ were added to the epoxy adhesive to investigate the residual strength of the joints after heat activation. Initial trials showed that $3 \%$ wt EG was sufficient to promote complete separation of the joints. The disbonding effectiveness (Eqn.1) curves are given in Fig. 10. A similar trend to the PU adhesive was found, namely that a low (<3\%wt) EG content had little effect on initial joint strength but had high disbonding effectiveness. Compared to the PU, a more sudden change in residual strength was observed for the epoxy which exhibited a decrease in joint strength from $21.41 \mathrm{MPa}$ to zero from $1 \%$ wt to $3 \% \mathrm{wt}$ of added EG. It was also noted that the residual joint strength of pure epoxy was reduced by $30 \%$, compared with a loss of $73 \%$ for the PU adhesive.

\subsection{Effect of additive on Environmental Durability of the Joints}

\subsubsection{Environmental Conditioning on SLJ}

The first stage of the environmental conditioning test was completed for epoxy joints with $3 \%$ wt of EG content. Test specimens were subjected to water immersion at $55^{\circ} \mathrm{C}$ for 90 days. The pure epoxy joints lost approximately $30 \%$ of their initial lap shear strength, whilst the joints containing $3 \%$ wt of added EG lost around $22 \%$ of their initial value (Table 3 ).

\subsubsection{Crack Propagation in Wedge Tests}

Wedge cleavage specimens were immersed in water at $55 \mathrm{C}$ to examine the crack growth in joints made with PU and epoxy adhesives, with and without EG additives. Crack growth data was measured over a 7-day period (Fig. 11). 
Generally, the epoxy had superior crack resistance compared to the PU adhesive. The crack growth in the epoxy specimens stagnated within 24 hours whereas it continued to grow in the PU specimens, leading to near separation after 7 days. The crack length of the epoxy test joints containing EG was 35\% less than for the pure epoxy joints, except for the initial and final state. This may be attributed to the different locus of failure of the joints. The wedge specimens with EG failed more cohesively than the pure epoxy ones, in which a mixed mode of adhesion and cohesive failure was observed.

To estimate the fracture toughness of the joints, the adhesive fracture toughness was calculated using Equation 2. This is also known as the mode 1 strain energy release rate, $\mathrm{G}_{1}$. Both adhesive types exhibited remarkably similar initial fracture toughness (Fig. 12).

\subsection{Evaluation of Disbonded Joint Fracture Surfaces with SEM}

SEM images were obtained to characterise the microstructural morphology of the fracture surfaces and EG particles. Fig. 13 (a) (b) shows the SEM micrographs of PU / EG 15\%wt before heat activation. Unexpanded EG can be identified in (a) and (b) as dark flakes embedded in the PU matrix. The stacked arrangement of the EG flakes is visibly parallel to the substrate. The bubbles in the image are small air pockets trapped inside the adhesive during fabrication. 
Fig.13 (c), (d) shows the SEM image of the joint after EG activation. The vermicular structure of the expanded graphite is visible, as well as the gaps and fractures created by the expansion of the EG.

Fig.14 shows the pure PU bondline after exposure to a temperature of $235^{\circ} \mathrm{C}$, to compare the microstructure of PU adhesive without and with EG inclusion (Fig. 13). An aerated sponge-like structure was found for PU, which explains the expansion that is responsible for the loss in strength. Comparing Fig.13 (a) and 14 (a), it can be seen that the voids have expanded significantly after heat treatment. The boundary of the distended EG and adhesive is clear, which can either be explained by a lack of adhesion between the graphite and the PU, or by exfoliation of the superficial layers of the graphite flakes during the destructive testing.

\section{Discussion}

\subsection{Disbonding performance of Adhesive/ EG systems}

The minimum effective concentration of EG within the bondline was evaluated from the residual strengths when lap joints were subjected to a temperature of $235^{\circ} \mathrm{C}$. Figures 15 and 16 show the values for initial and residual joint strengths with EG concentrations between 0 and 5\%wt.

Joints made with the PU adhesive exhibited a $73 \%$ loss in strength, even when no EG was present, indicating that temperature alone was responsible for weakening the joints. Joints made with the epoxy adhesive exhibited a corresponding loss of less than $14 \%$. Figures 15 and 16 show that residual 
strengths fell to zero for an EG concentration of $5 \%$ wt in the PU joints and $3 \%$ wt in the epoxy joints.

This result is very encouraging when compared to the disbonding performance associated with the incorporation of physical and chemical foaming agents into bondlines. Banea et al [9], McCurdy et al [5] and Lu et al. [3] all reported that addition levels of around $15 \%$ wt are necessary to trigger disbonding with epoxy adhesives, and that no addition level was effective with PU adhesives. The superior effectiveness of the EG may be attributed largely to the greater expansivity of this material.

\subsection{Disbonding mechanism of Adhesive/ EG systems}

\subsubsection{Adhesive degradation during heating}

Adhesive degradation as a direct result of heating was observed for both adhesives, but it was much more significant for the PU. The results showed a decrease in strength of $73 \%$, for SLJs made with pure PU and $0.2 \mathrm{~mm}$ bondline, and even of $90 \%$ when a $1 \mathrm{~mm}$ bondline was used. A less significant loss in strength occurred for the epoxy adhesive, of only $22 \%$. Arguably, adhesive degradation during heating could be regarded as the primary cause for disbonding in the case of PU adhesives, although total disbonding only took place in the presence of EG.

\subsubsection{Differences in the thermal expansion coefficient}

It is possible that another secondary cause for disbonding is the difference in thermal expansion during the changes in temperature, between the aluminium 
substrates and the adhesive used. Adams et al. [36] noted a difference in thermal expansion coefficients of between 7 to 8 times, which will further weaken the integrity of the joint and contribute to the disbonding performance.

\subsubsection{Crack propagation}

Crack propagation within the bondline until total rupture was identified as the main explanation of disbonding for adhesive/EG systems. As the graphite is an inert material, chemical reaction between the additive and the adhesive can be ruled out. Therefore, the mechanism of rupture can only be explained by the internal mechanical stresses generated by the expansion of the graphite flakes within the adhesive layer.

The tendency of the bondline is to oppose an increase in volume but, once heat is added into the system, a dramatic increase in pressure generated by the interstitial gases will take place. As soon as the inner pressure is sufficient to affect the adhesive matrix, micro-cracks will appear which will weaken the bondline, allowing for an incipient expansion of the EG. Total rupture of the bondline will occur if the inner pressure of the expanding EG is sufficient to overcome the adhesive's resistance, and the initial micro-cracks will evolve and meet inside the bondline. As noted by Kishi et al [11], a low modulus or tough polymer matrix will be able to absorb more expansion than a higher modulus or less tough material.

The test results show that a concentration of $3 \%$ wt EG added to a PU adhesive was not sufficient to generate total disbonding of a joint with an initial strength of $19.5 \mathrm{MPa}$. Tests for residual strength revealed an average 
value of $0.6 \mathrm{MPa}$ in this case. Conversely, total disbonding was achieved for the epoxy based SLJ's made with the same EG concentration, even though the initial strength value was significantly higher (26.6 MPa).

\subsection{Effect of environmental conditioning on lap joint strength}

Almost $30 \%$ of the initial control strength of lap joints made with epoxy adhesive was lost after a period of immersion for 90 days in tap water at $55^{\circ} \mathrm{C}$, when compared with the lap shear strength of unconditioned joints. This result for joints without any EG was slightly surprising, but the reasons for the strength reduction may be attributed to loss of adhesion and plasticisation of the bondline.

A similar comparison was made between joints incorporating 3\%wt EG within the adhesive and joints made with pure adhesive. It was found that only $22 \%$ of the initial control strength was lost after the same period of water immersion. Visual bondline analysis revealed that some of the EG flakes were only partially covered inside the adhesive/ additive matrix, allowing the water to diffuse further into the bondline and leading to bondline plasticisation.

This result is very positive when compared to previous work using physical and chemical foaming agents, where previous research has resulted in very significant losses in joint strength. McCurdy et al [5] reported reductions in joint strength of 25 to $71 \%$ for the same epoxy system. Lu et al [3] attributed loss of joint strength mostly to a loss of adhesion at the thermoplastic microsphere/epoxy interface and demonstrated that one way to prevent it was to surface treat the microspheres carefully prior to their incorporation into the bondline. 


\subsection{Fracture toughness of bondlines}

Wedge cleavage joints were used to assess the effect of EG on bondline fracture toughness. The general finding was that the effect of a $5 \%$ wt EG addition level to the PU had little or no effect; a value of $G_{1}=2.9 \mathrm{MJ} / \mathrm{m}^{2}$ was recorded in both cases. However, the effect of a 3\%wt EG addition level to the epoxy led to a $25 \%$ increase in the strain energy release rate, rising from $\mathrm{G}_{1}=2.8 \mathrm{MJ} / \mathrm{m}^{2}$ to $\mathrm{G}_{1}=3.5 \mathrm{MJ} / \mathrm{m}^{2}$. It is assumed that a crack is arrested by the presence of the additive. A similar strengthening effect was reported by Lu [3], in a bulk test, and by Banea [9] in a double cantilever test, where a low percentage of particle additive was found to increase the strength and fracture toughness of the adhesive system.

\subsection{Assessment of the durability of bonded assemblies}

Wedge cleavage joints, immersed in water at $55^{\circ} \mathrm{C}$, were used to assess the effect of EG on joint durability. A comparison between the durability assessment results for PU and Epoxy based matrices showed that the PU based matrices were much more sensitive to the accelerated ageing process as compared with the epoxy ones. Several observations can be made from the results:

- The crack length in PU developed more rapidly, being close to total failure after only 72 hours

- The development curve for PU shows a quasi - constant crack propagation in time, as opposed to the epoxy crack development which shows a higher increase in the first 6 hours and then a tendency to grow at a much lower rate 
- The difference in crack propagation between the epoxy adhesive (aprox. 65\% higher for pure Epoxy, after 6 hours) could be explained by the different initial locus of failure of the pure epoxy joints, which exhibited adhesion failure (visually). Given that a silane primer was involved in manufacturing the joints, the adhesion failure could have taken place between the primer and the adhesive, or between the primer and the substrates. Further work could determine the exact locus of failure in this case.

- A comparison between the pure PU and PU/EG adhesives revealed an increase in crack propagation of around 6\% throughout. No significant differences in crack propagation development type were observed.

- A similar comparison between joints made with epoxy adhesives is difficult because of the different locus of failure. However, it can be observed that, in the case of epoxy/EG $3 \%$ wt joints, after an initial increase in length during the first 6 hours, the crack propagation stopped almost completely, meaning that the presence of EG flakes within the bondline arrested the crack. It is possible that the rounded voids surrounding the flakes in the bondline act as stress dissipation agents. Initial investigation of crack tips under the microscope suggested this as a possible explanation

Strain energy release rate values were plotted as a function of time and exposure condition. Again, the epoxy/EG 3\%wt joints demonstrate increased resilience when subjected to accelerated aging. Initial fracture energy values are consistent with literature values for epoxies (typically 1-10 $\mathrm{MJ} / \mathrm{m}^{2}$ ). 


\section{Conclusions}

It is possible to use small additions of low cost, thermally activated, expandable graphite (EG) within commercially-available adhesives for the purpose of dismantling bonded joints at $235^{\circ} \mathrm{C}$. No prior preparation of the EG was required before mixing with the adhesives.

The effective EG percentage addition levels were found to be: $5 \% \mathrm{wt}$ for $2 \mathrm{~K}$ Polyurethane SikaForce $7888 \mathrm{~L} 12$, and $3 \% \mathrm{wt}$ for $2 \mathrm{~K}$ Structural Epoxy $3 \mathrm{M}^{\mathrm{TM}}$ Scotch-Weld ${ }^{\mathrm{TM}}$ EC-9323 B/A.

The failure mechanism was identified as the internal mechanical stresses developed within the matrix by the thermally activated expansion of EG. No chemical reaction between the adhesives and the EG was identified.

The bonded joint cure temperature of $90^{\circ} \mathrm{C}$ had no significant effect on the material properties of the adhesive/EG combinations investigated.

There was no significant loss in lap shear strength, or fracture toughness, of the joints made with EG as compared with pure adhesive joints.

Environmental conditioning, representative of extreme in-service conditions, revealed only a $3.6 \%$ decrease in strength due to the presence of EG within the epoxy adhesive. This further demonstrates the inert characteristics of the EG, chemically neutral to both the adhesive and water. The decrease in 
tensile lap shear strength could be explained by water infiltration between the adhesive and the EG flakes at the interfacial layers of the bondline.

During environmental conditioning, the presence of $3 \%$ wt EG within an epoxy adhesive had a positive toughening effect, reducing the rate of crack growth when compared to corresponding joints with pure adhesive. For the PU adhesive, the presence of 5\%wt EG showed a slight increase in crack propagation rate.

\section{Acknowledgements}

This research was part-funded by the UK Engineering and Physical Sciences Research Council (EPSRC), grant ref EP/I038616/1. The expanding graphite particles, and suggestions for their use, were provided by Professor Sato of the Tokyo Institute of Technology.

\section{References}

[1] Hutchinson, A.R., Winfield, P.H. and McCurdy, R.H., Adv. Eng. Mater.12, 646-652 (2010).

[2] Lu, Y., Broughton, J. and Winfield, P.H., Int. J. Adhes. Adhes. 50, 119-127 (2014).

[3] Lu, Y., Broughton J., and Winfield, P.H., Int. J. Adhes. Adhes. 66, 33-40 (2016).

[4] Banea, M.D., da Silva, L.F.M., and Carbas, R.J.C., Int. J. Adhes. Adhes.59,14-20 (2015). 
[5] McCurdy, R.H., Hutchinson, A.R. and Winfield, P.H., Int. J. Adhes. Adhes. $46,100-113$ (2013).

[6] Rodrigues, D.D, Winfield, P.H. and Morrey, D., Mater. Sci. Forum. 765, 766-770 (2013).

[7] Loureiro, A. L., da Silva, L.F.M, Sato., C. and Figueiredo, M.A.V., J. Adhes. 86 (7), 765-778 (2010).

[8] Banea, M. D., da Silva, L. F. M. and Campilho, R. D. S. G. , J. Adhes. 90 , 16-40 (2014).

[9] Banea, M.D., da Silva, L.F.M., Carbas, R.J.C. and Campilho, R.D.S.G. , Int. J. Adhes. Adhes. 54, 191-199 (2014).

[10] Kishi H., Inada, Y., Imade, J., Uesawa, K., Matsuda, S. and Sato, C., J. Adhes. Soc. Jpn.42, 356-363 (2006).

[11] Kishi, H., Imade, J., Inada, Y., Sato, C., Matsuda, S.A.M. ,16 ${ }^{\text {th }}$ International conference on composite materials. Kyoto, Japan (2007).

[12] Furdin G., Fuel. 77, 479-485 (1998).

[13] Sorokina NE, Shornikova ON, Avdeev VV., J. Inorg. Mater. 43, 822-826 (2007).

[14] Hong, X and Chung, D., Carbon.9, 1-10 (2015).

[15] Leshin, V.S., Sorokina, N.E and Avdeev, V.V., J. Inorg. Mater. 40, 649_ 655 (2003).

[16] Kim, J., Seungkeun, O., Kim, H., Kim, B., Jeon,K-J. and Yoon, S-H. , Scientific Cooperations International Workshops on Engineering Branches. 89 (2014). 
[17] Zhu,Y.W., Murali, S., Stoller, M.D., Velamakanni, A., Piner, R.D. and Ruoff, R.S., Carbon. 48, 2118-2122 (2010).

[18] Gardelle, B., Duquesne, S., Vandereecken, P., Bellayer, S. and Bourbigot. S., Eur. Polym. J. 49, 2031-2041 (2013).

[19] Sever, K., Tavman, I.H., Seki, Y., Turgut, A., Omastova, M. and Ozdemir, I., Composite, 226-233 (2013).

[20] Fukushima, H., Drzal, L., Rook, B. and Rich, M., J. Therm. Anal. Calorim. 85, 235-238 (2006).

[21] Kim,S., Ryu, J. and Kato, Y. , App. Thermal. Eng. 66, 274-281(2014).

[22] Laachachi, A., Burger, N., Apaydin, K. and Sonnier, R., Polym. Degrad. Stab.117, 22-29 (2015).

[23] Focke, W. W., Muiambo, H., Mhike, W., Kruger, H. and Ofosu, O., Polym. Degrad. Stab. 100, 63-9 (2014).

[24] Focke, W.W., Badenhorst, H., Mhike, W., Kruger, H.J. and Lombaard, D., Therm. Acta. 584, 8-16 (2014).

[25] Liu,J., Zhang, Y., Peng, S., Pan, B., Lu, C., Liu, H., Ma, J. and Niu, Q. , Polym. Degrad. Stab. 121, 261-70 (2015).

[26] Chung, D.D.L., J. Mater. Eng. Perform. 9, 161-3 (2000).

[27] Afanasov, I., Shornikova,O., Acdeec, V., Lebedev, O., Tendeloo, G. and Matveev, A., Carbon. 47, 513-518 (2009). 
[28] Zhou, K., Shi, Y., Jiang, S., Song, L., Hu, Y. and Gui, Z., Mater. Res. Bull. 48, 2985-2992 (2013).

[29] Zu, C., Li, L., Qie, L. and Manthiram, A. , J. Power. Sour. 284, 60-67 (2015).

[30] Alashdan, A., Mayyas, A.T. and Al-Hallaj, S., J. Mater. Process. Technol. 210,174-179 (2010).

[31] Wei, Y., Jin, Q and Ren, T., Solid-State Elec. 63(1), 76-82 (2011).

[32] Chun, J.H., Jo, D. H., Kim, S.G., Park, S.H., Leem C-H., Lee, E. S., Jyoung, J-Y. and Kim, S. H. Renewable Eneg. 58, 28-33 (2013).

[33] Serna, P.O., Sanchis, M.J., Gomez. C.M. and Cantarero, A., Polym. Int. 64, 284-292 (2015).

[34] Kang, W-S., Rhee, K.Y. and Park, S-J., Composites Part B. 94, 238-244 (2016).

[35] Neto, J.A.P.B, Campilho, R.D.S.G and Silva, L.F.M., Int. J. Adhes. Adhes. 37, 96-101 (2012).

[36] Adams, R., Comyn, J., and Wake W.C. Structural Adhesive Joints in Engineering, (Chapman \& Hill, London, 1997). 2nd ed., Chap. 5, pp.194-195. 
Fig. 1. The flaky lamellar structure of natural flake graphite at (a) 60X and (b) 250X magnification
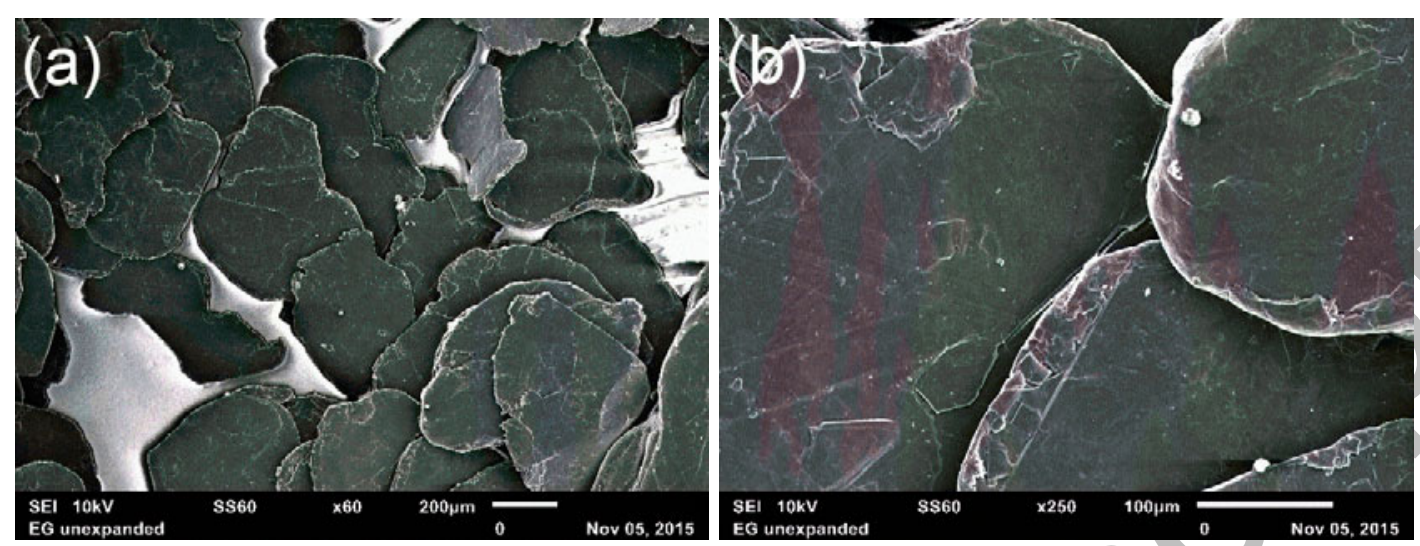
Fig. 2. Graphite flakes before (a) and after expansion / exfoliation (b).

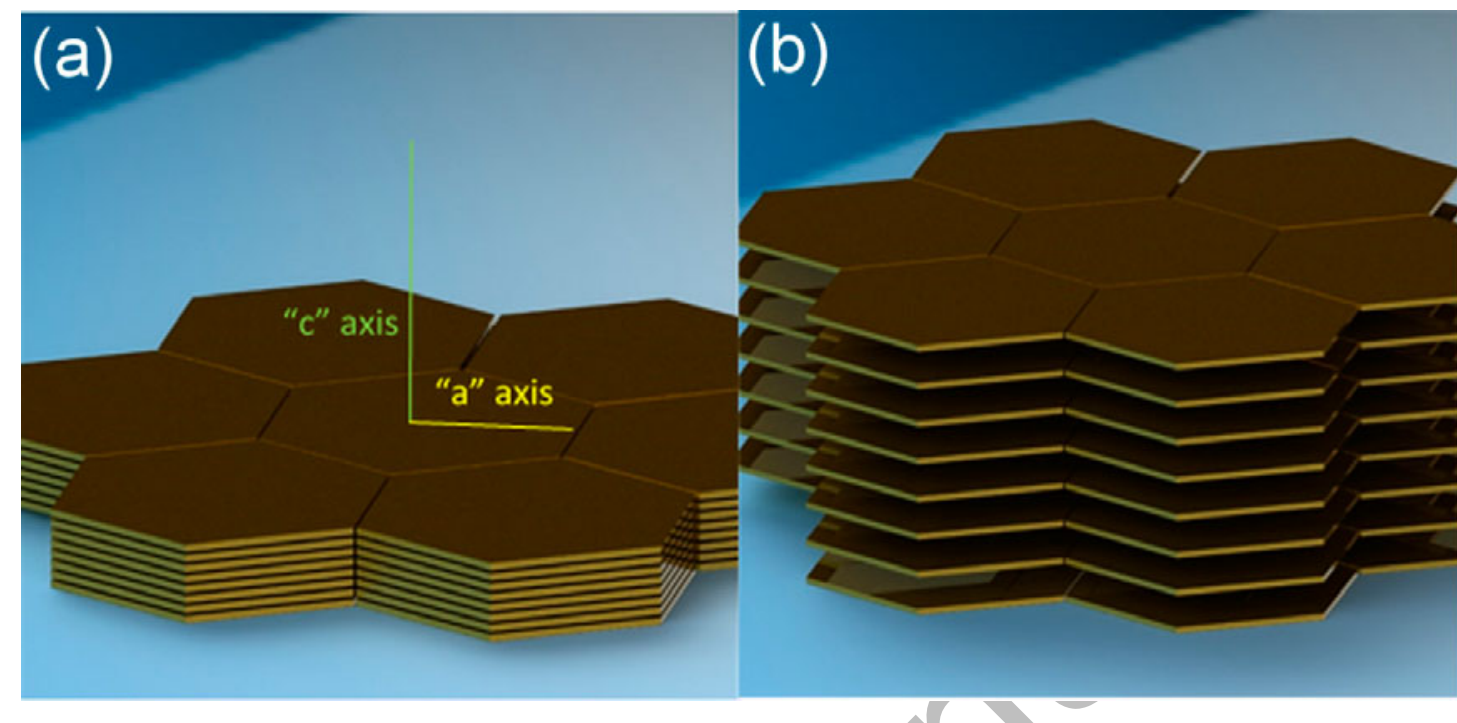


Fig. 3. Graphite flakes after heat triggered expansion/exfoliation at (a) $60 \mathrm{X}$ and (b) 250X magnification
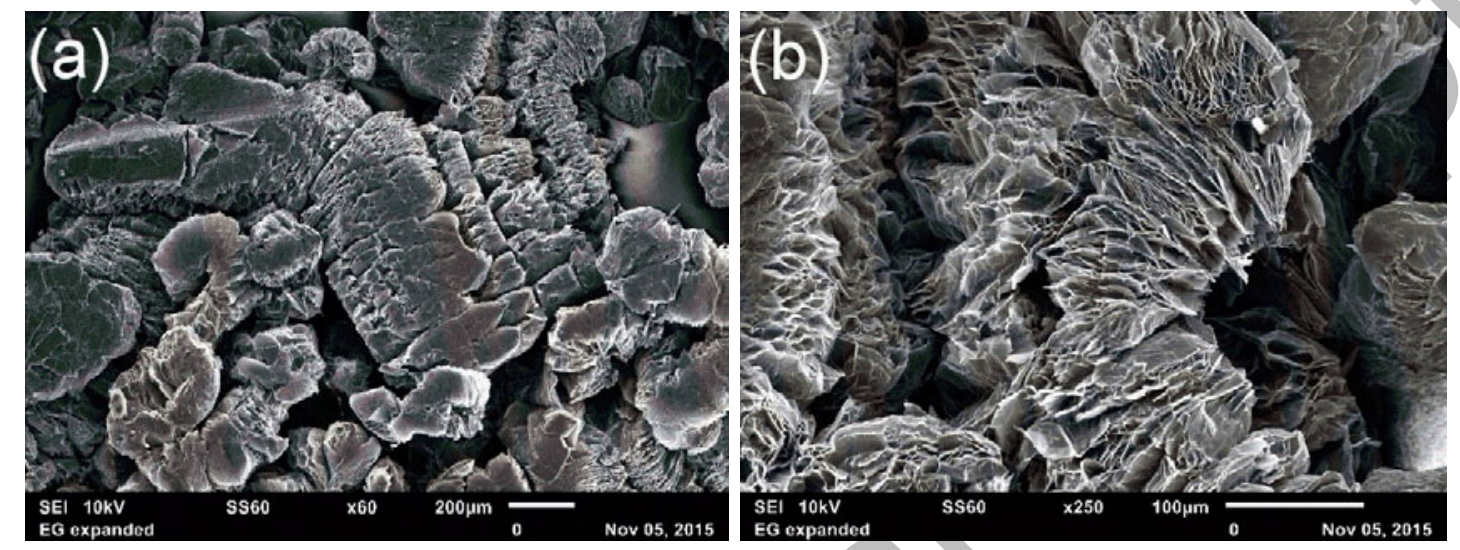
Fig. 4. Flow chart of experimental programme
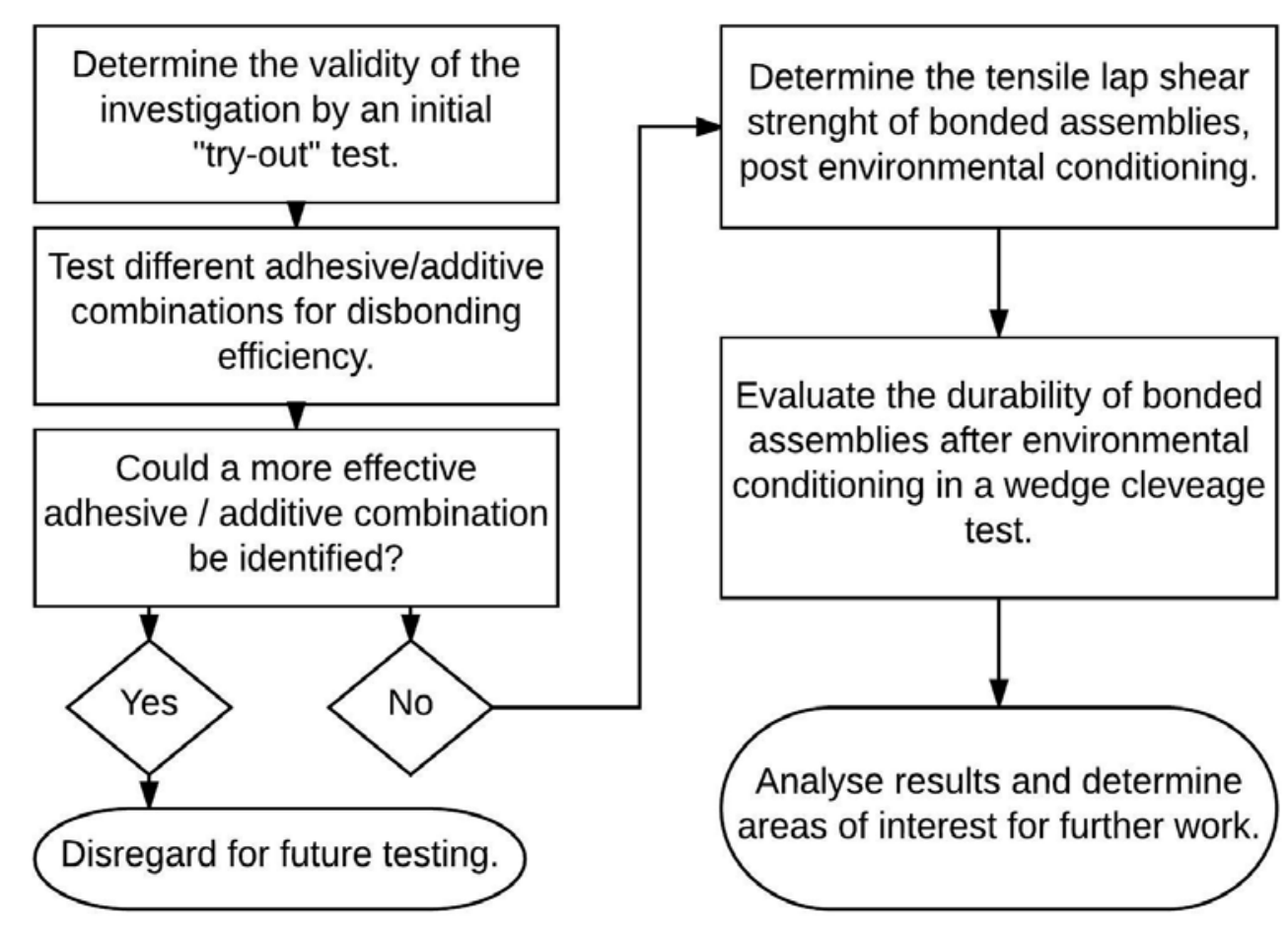
Fig. 5. Expansion volume of EG SYZR 501 according to temperature (Source: SanyoCorp Datasheet)

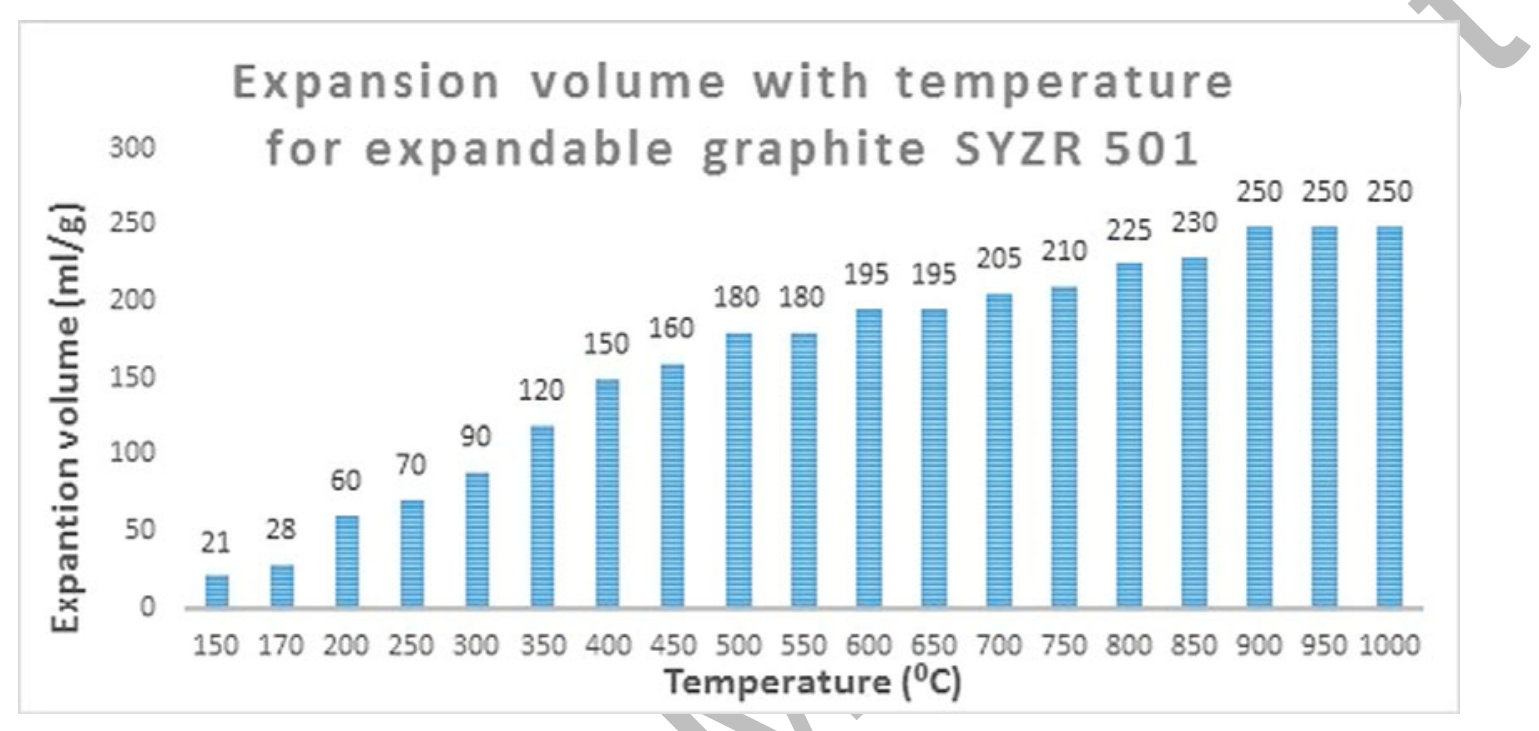


Fig. 6. Geometry of single lap-shear test specimen (a: Gripped area b:

Bonded area)

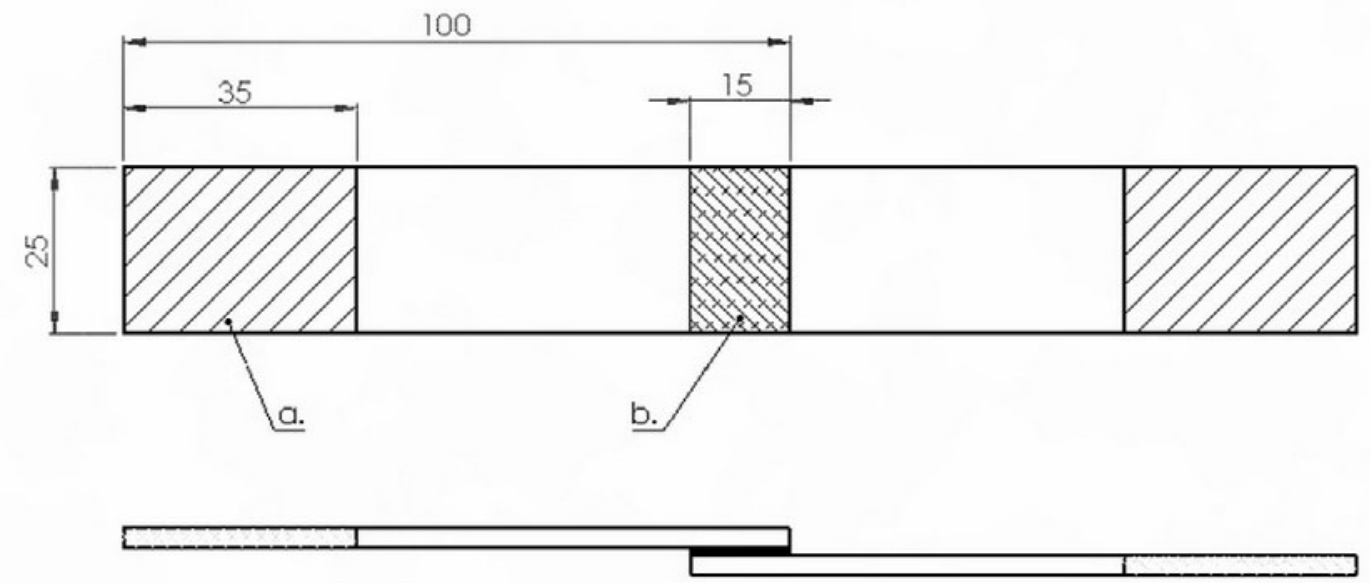


Fig. 7. Geometry of wedge cleavage test specimen

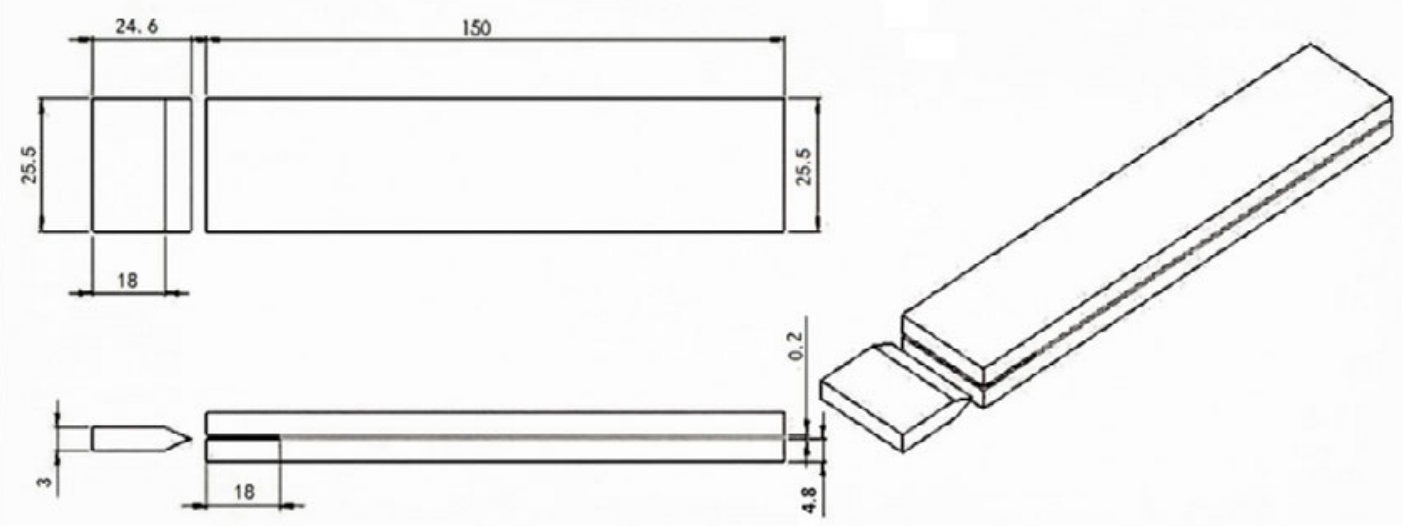


Fig. 8. Joint separation with EG activated in PU
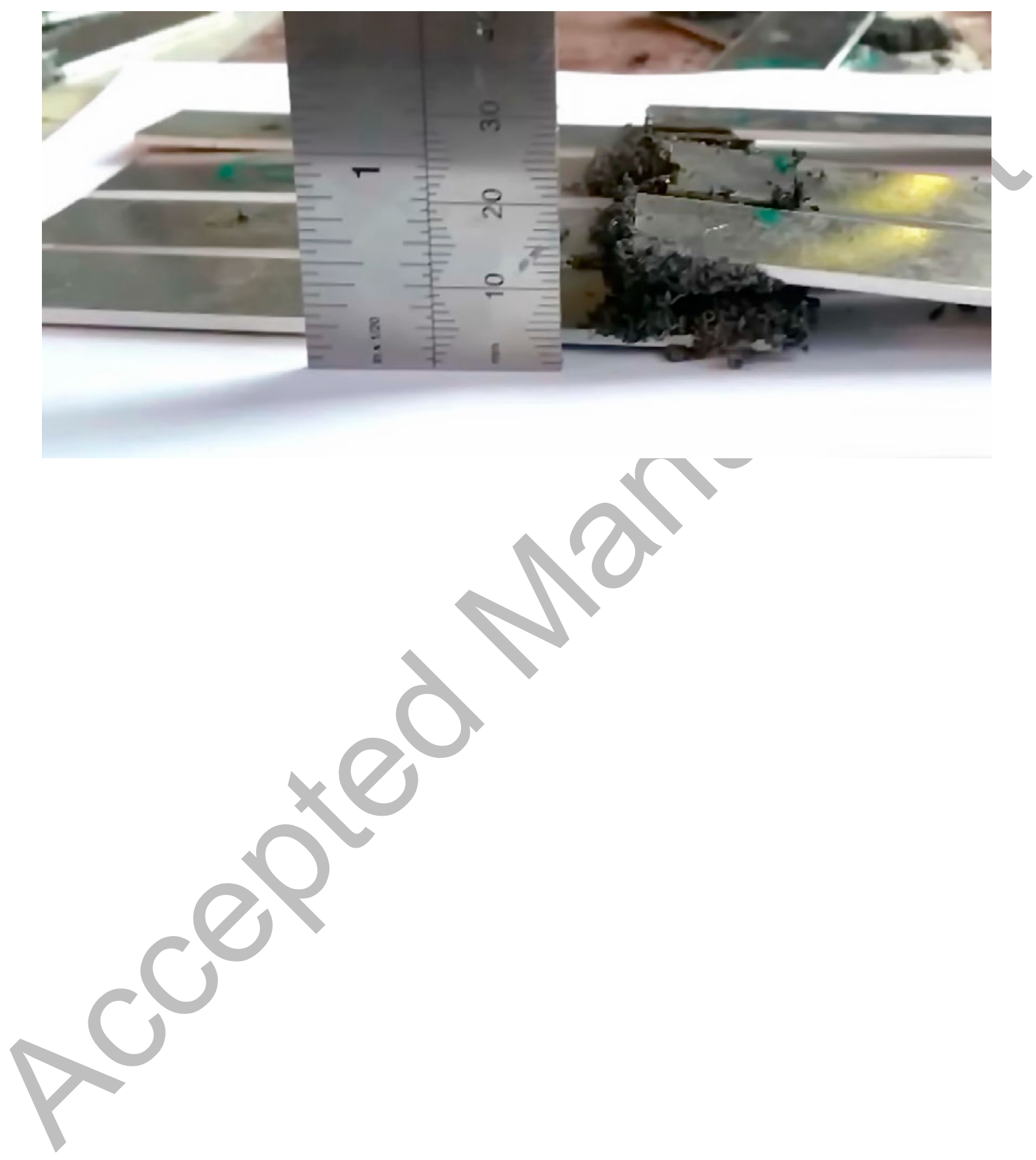
Fig. 9. Disbonding effectiveness as a function of \%wt EG content in PU

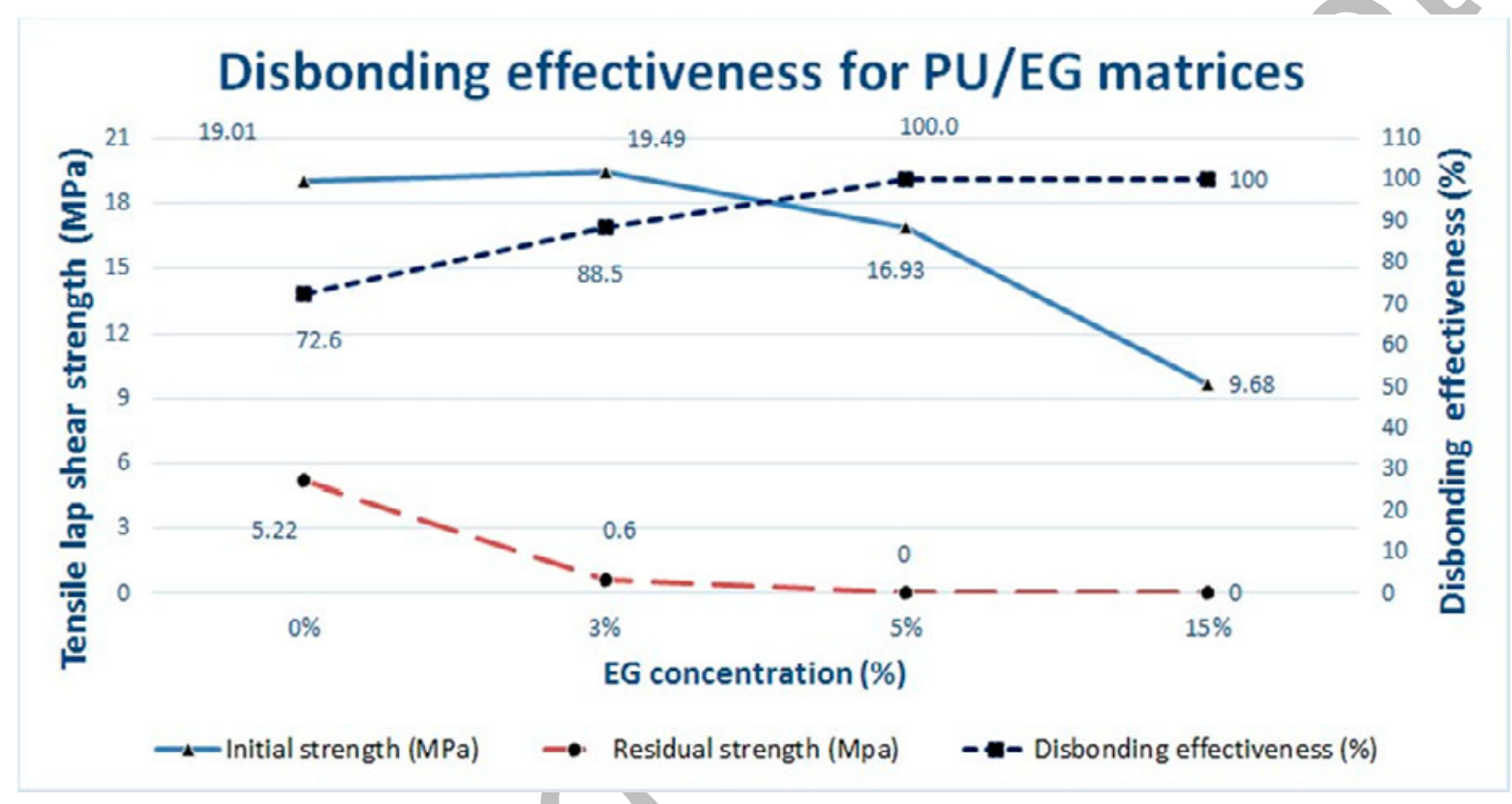


Fig. 10. Disbonding effectiveness as a function of \%wt EG content in epoxy

\section{Disbonding Effectiveness for Epoxy/EG matrices}

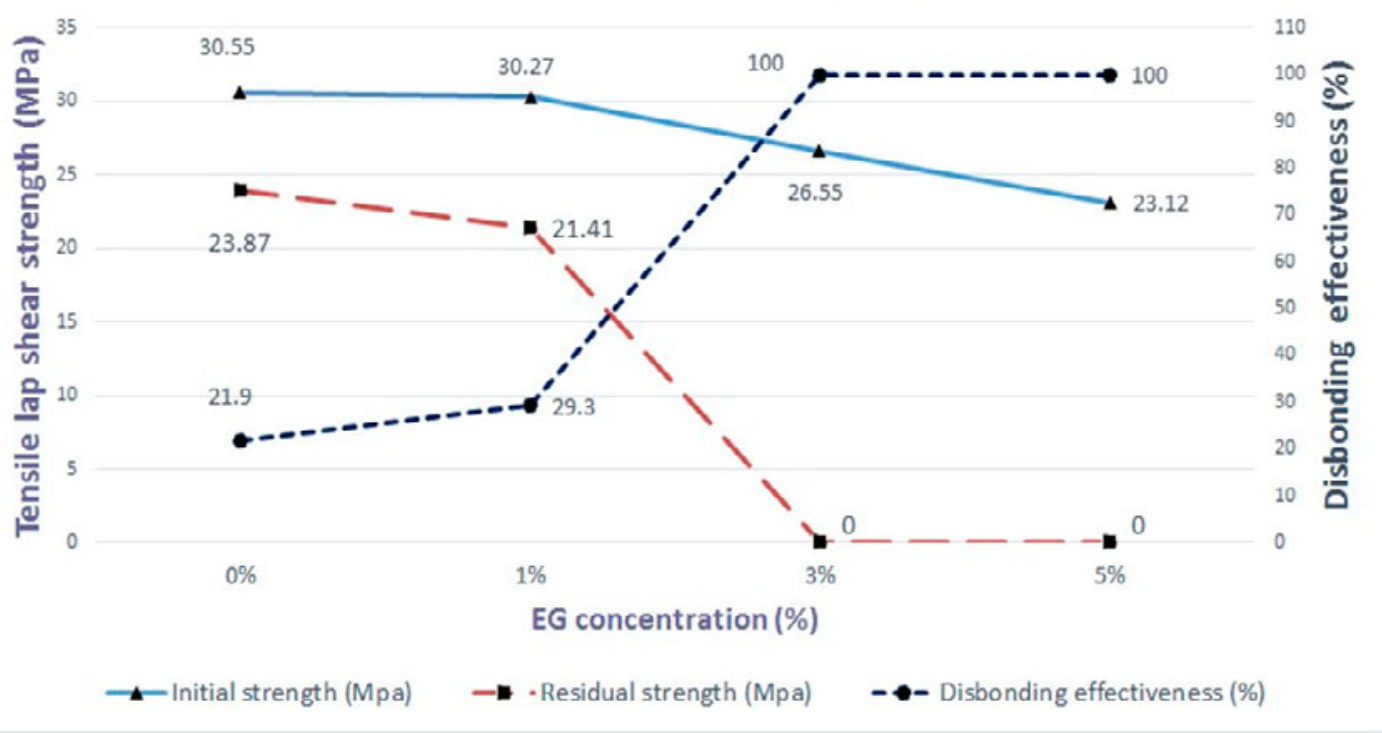


Fig 11. Crack propagation with time for epoxy and PU joints

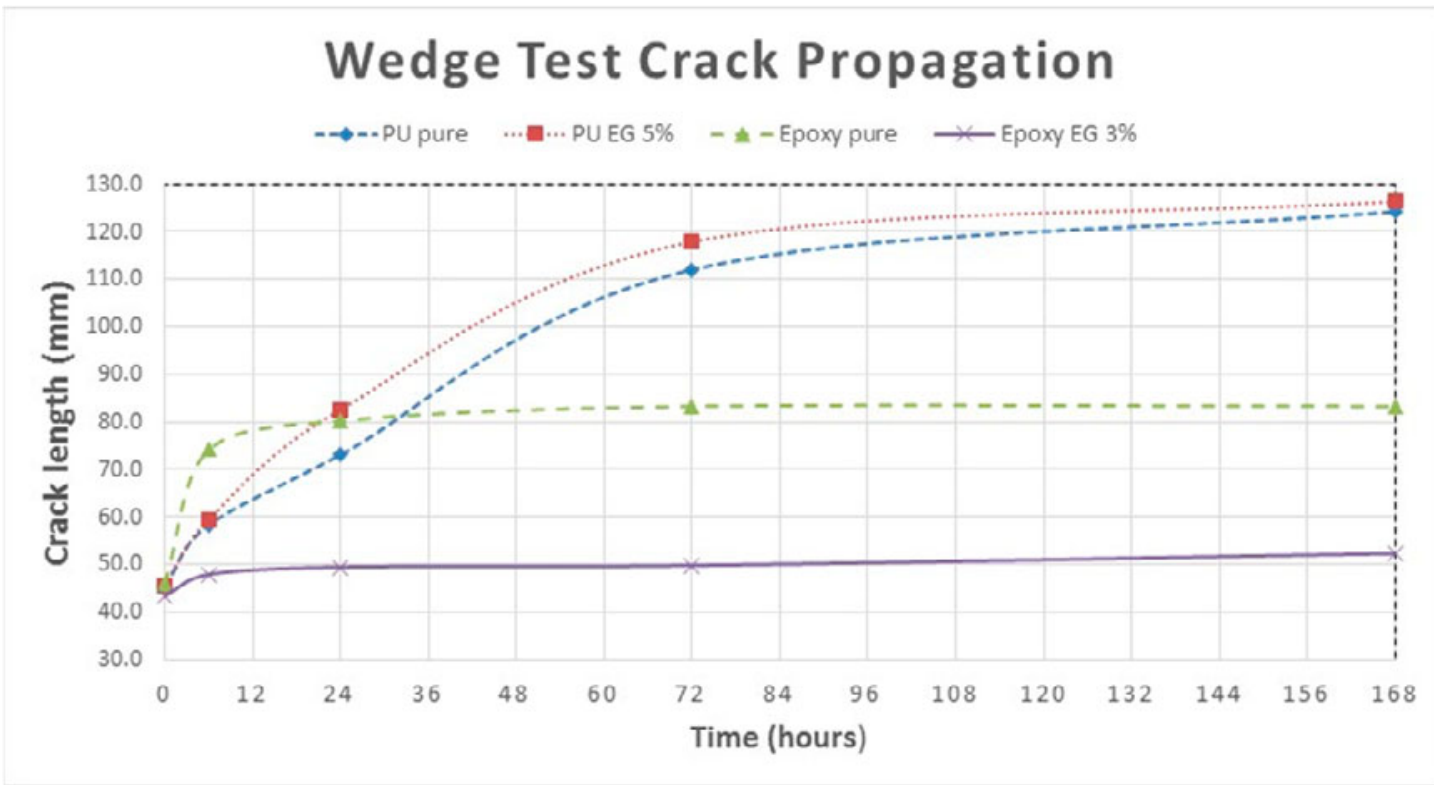


Fig. 12. Strain rate release rate of epoxy/EG system in wedge test

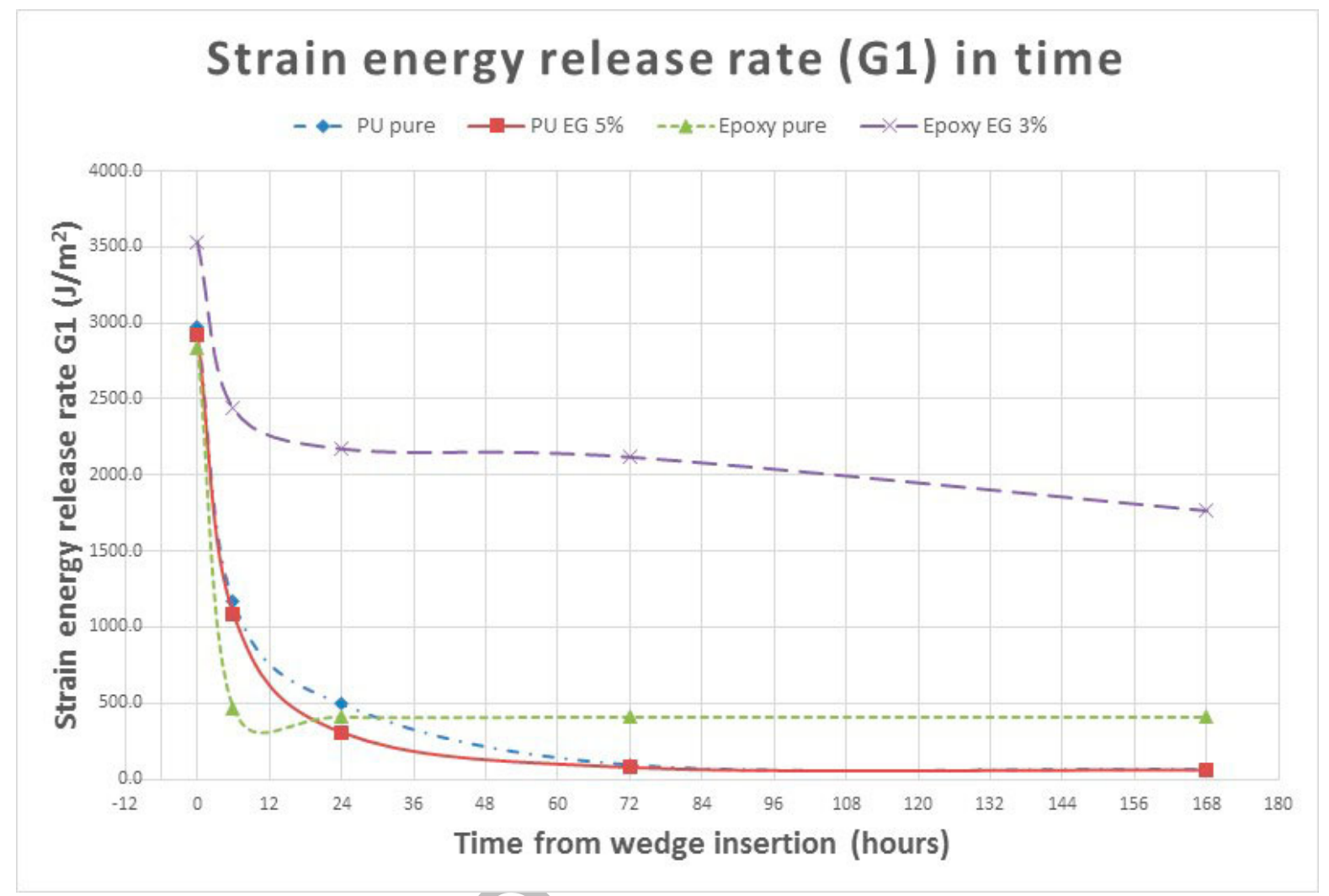


Fig.13. PU with 15\%wt EG inclusion before (a), (b) and after (c), (d) activation at 60X (a), (c) and 250X (b), (d) activation magnification

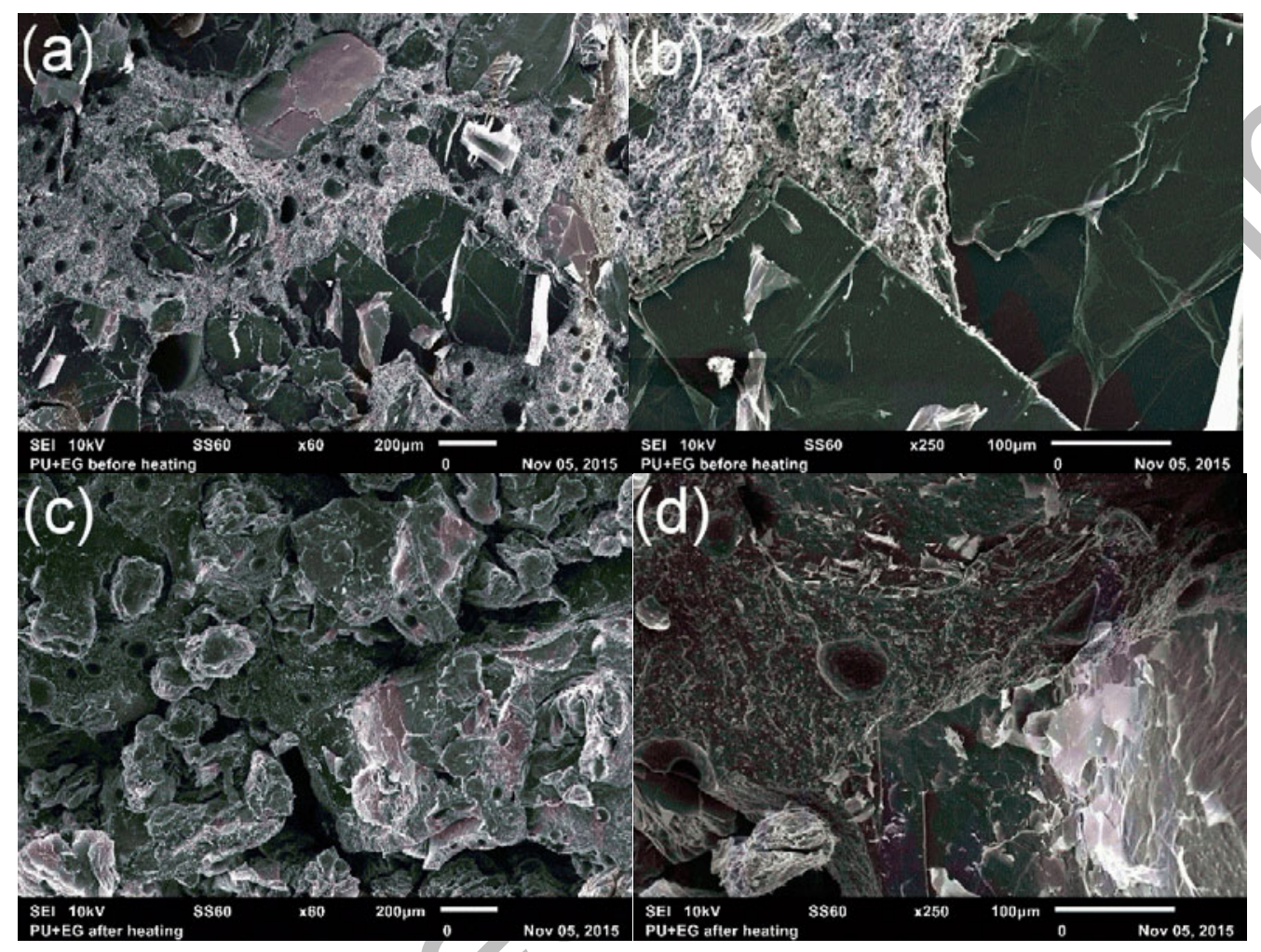


Fig. 14. Pure PU adhesive after exposure to $235^{\circ} \mathrm{C}$ at (a) $60 \mathrm{X}$ and (b) $250 \mathrm{X}$ magnification
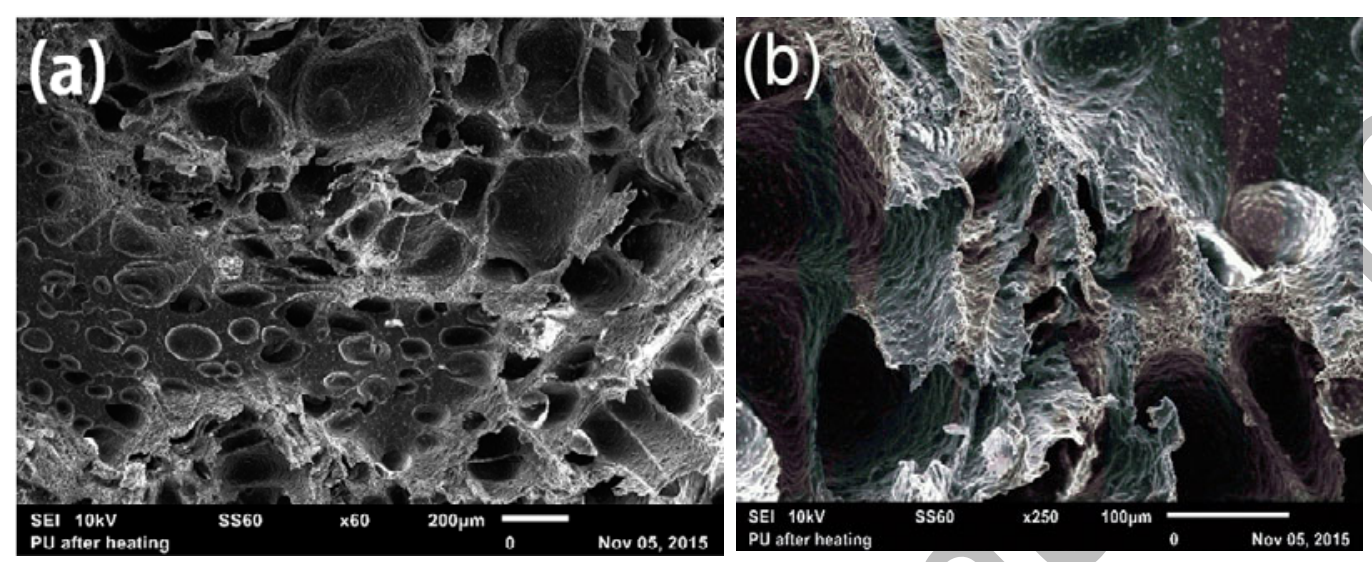
Fig. 15. Initial and residual tensile lap shear strength values for PU adhesive, before and after heat activation

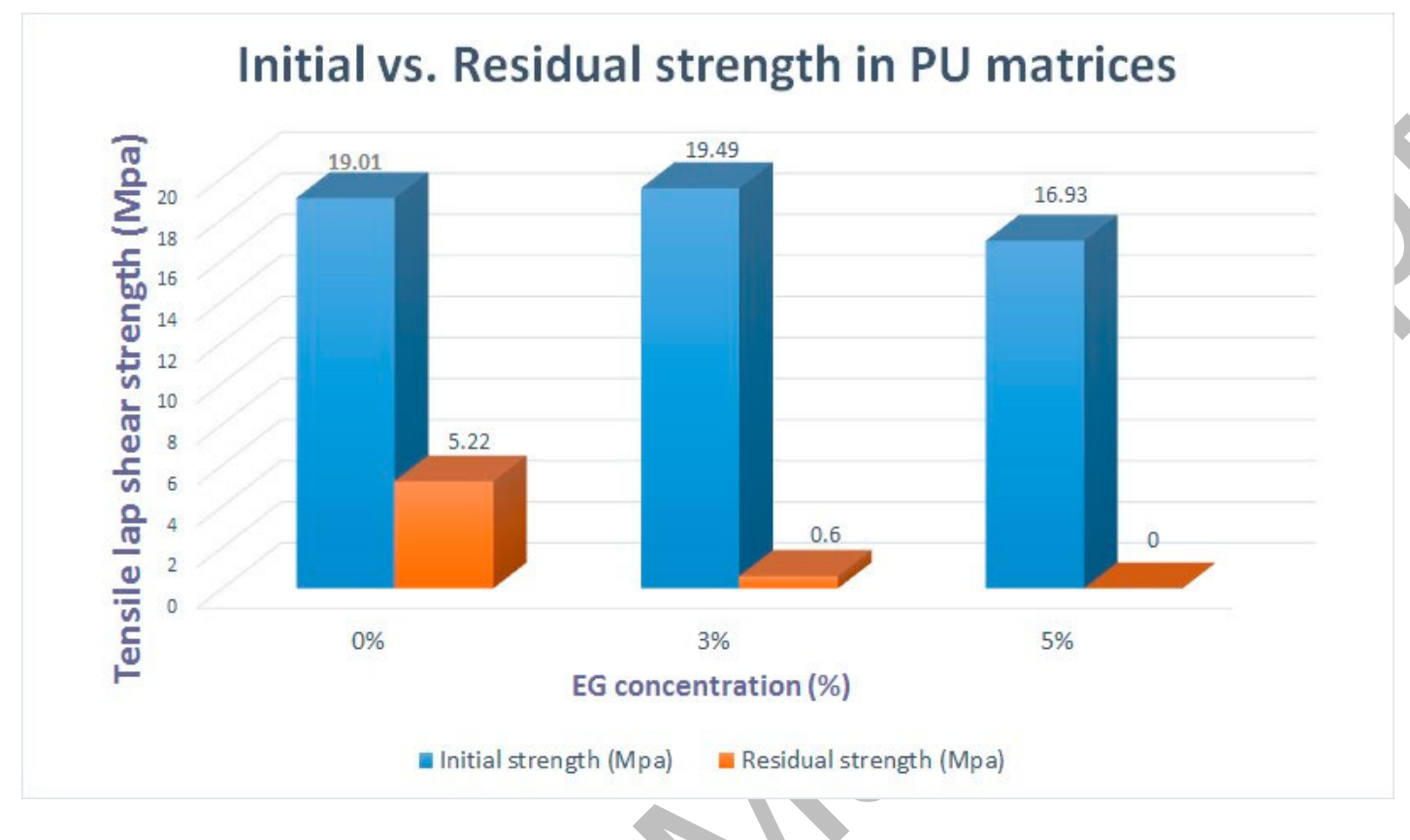


Fig. 16. Initial and residual tensile lap shear strength values for epoxy adhesive, before and after heat activation

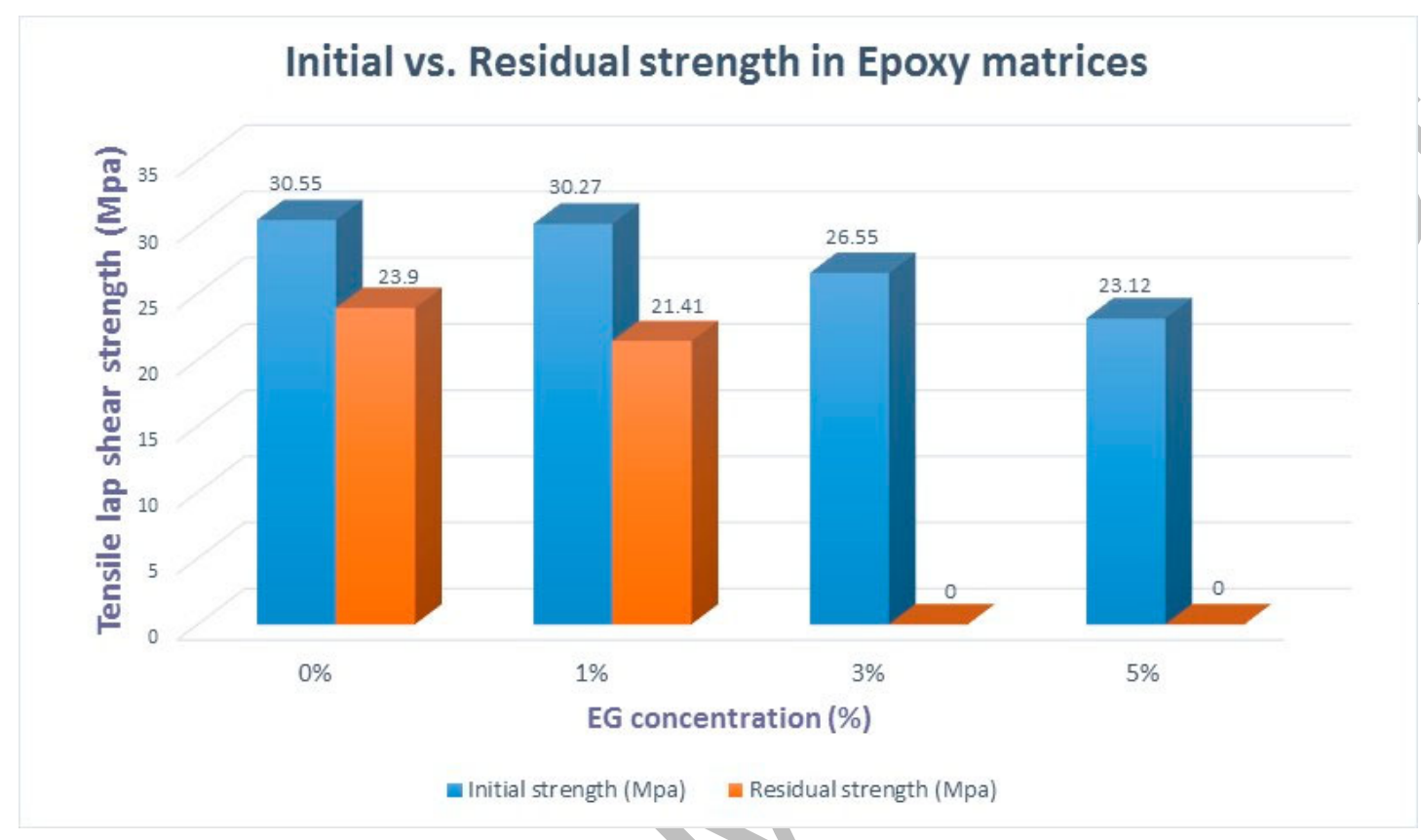


Table 1 Mechanical properties of epoxy and PU adhesives [3, 35]

\begin{tabular}{l|cc}
\hline Mechanical Properties & Scotch-Weld ${ }^{\mathrm{TM}}$ & SikaForce 7888 \\
& EC-9323 Epoxy & Polyurethane (PU) \\
\hline Ultimate Tensile Strength (MPa) & 41 & 29 \\
Tensile Modulus (GPa) & 2.1 & 1.9 \\
$\operatorname{Tg}\left({ }^{\circ} \mathrm{C}\right)$ & 94 & 40 \\
\hline
\end{tabular}


Table 2 Tensile properties of aluminium EN AW-6082 T6 substrates

\begin{tabular}{l|cc}
\hline Tensile Properties & Mean Value & Standard Deviation \\
\hline Ultimate Tensile Strength (MPa) & 350 & 6.8 \\
Yield Stress (MPa) & 310 & 3.8 \\
Strain at Yield (\%) & 1 & 0.01 \\
Young's Modulus (Gpa) & 78 & 5.8 \\
\hline
\end{tabular}


Table 3 Tensile strength of environmentally conditioned epoxy joints

\begin{tabular}{l|lc}
\hline Lap shear strengths (MPa) & Pure Epoxy & Epoxy / EG 3\%wt \\
\hline Control & $30.6(0.45)$ & $26.6(0.40)$ \\
After water immersion & $21.4(1.76)$ & $20.7(0.87)$ \\
\multicolumn{2}{|c}{ Standard deviation shown in parentheses }
\end{tabular}

